\title{
TENOR risk score predicts healthcare in adults with severe or difficult-to-treat asthma
}

\author{
M.K. Miller*, J.H. Lee*, P.D. Blanc ${ }^{\#}$, D.J. Pastaף, S. Gujrathi*, H. Barron*, \\ S.E. Wenzel ${ }^{+}$and S.T. Weiss ${ }^{\S}$ for the TENOR Study Group
}

ABSTRACT: The aim of the present study was to predict which patients with severe or difficult-totreat asthma are at highest risk for healthcare utilisation can be predicted so as to optimise clinical management.

Data were derived from 2,821 adults with asthma enrolled in The Epidemiology and Natural History of Asthma: Outcomes and Treatment Regimens (TENOR) study. Multiple potential predictors were assessed at baseline using a systematic algorithm employing stepwise logistic regression. Outcomes were asthma-related hospitalisations or emergency department (ED) visits within 6 months following baseline.

Overall, 239 subjects (8.5\%) reported hospitalisation or ED visits at follow-up. Predictors retained after multivariate analysis were as follows: younger age; female sex; non-white race; body mass index $\geqslant 35 \mathrm{~kg} \cdot \mathrm{m}^{-2}$; post-bronchodilator per cent predicted forced vital capacity $<70 \%$; history of pneumonia; diabetes; cataracts; intubation for asthma; and three or more steroid bursts in the prior 3 months. A final risk score derived from the logistic regression model ranged from 0 18 and was highly predictive (c-index: $\mathbf{0 . 7 8}$ ) of hospitalisation or ED visits. This tool was re-tested in a prospective validation using outcomes at 12- to 18-months follow-up among the same cohort (c-index: 0.77).

The risk score derived is a clinically useful tool for assessing the likelihood of asthma-related hospitalisation or emergency department visits in adults with severe and difficult-to-treat asthma.

KEYWORDS: Asthma, epidemiology, healthcare use, predictor, risk score, TENOR

$\mathbf{N}$ early 300 million persons worldwide suffer from asthma [1]. The high economic costs of adult asthma include direct medical costs, such as emergency department (ED) visits and hospital admissions, as well as indirect costs associated with loss of productivity [1-3]. Moreover, higher asthma costs have been consistently associated with increasing asthma severity [4-6]. In 2004, acute hospital care (ED and inpatient care) accounted for $31 \%$ of the $\$ 11.5$ billion in direct costs associated with asthma in the USA [7]. Earlier studies suggested that acute hospitalisation accounted for $>50 \%$ of direct costs for asthma [8,9].

Greater asthma severity, race/ethnicity, sex and psychosocial variables have been demonstrated to be related to subsequent asthma-related emergency care and hospitalisation (as markers of morbidity) and to mortality [10-21]. Assessment of morbidity and mortality risk can influence clinical decision-making regarding targeted asthma pharmacotherapy and other asthma-related medical care. Early identification of patients at increased risk of adverse outcomes, as well as better understanding of specific interventions of benefit, would allow a proactive approach for asthma care, for example, modifications in targeted asthma pharmacotherapy. Indeed, intervention programmes for patients at increased risk of asthma exacerbations have been shown to reduce subsequent healthcare use $[22,23]$. Despite the potential advantages of a standardised approach to risk assessment in asthma, systematic evaluations of predictors for healthcare use in a large group of severe or difficult-to-treat asthma patients are limited.

The aim of the present study was to develop and test a simple, integrated predictive tool to aid in the clinical identification of patients at increased risk of asthma-related hospitalisation or ED visits. This was carried out using data from severe and difficult-to-treat adult asthma patients enrolled in The Epidemiology and Natural History of Asthma: Outcomes and Treatment Regimens (TENOR) study.
AFFILIATIONS

*Genentech, Inc.

\# University of California,

"Ovation Research Group, San

Francisco, CA

${ }^{+}$National Jewish Medical and Research Center, Denver, CO, and

${ }^{\S}$ Channing Laboratory, Brigham and Women's Hospital, Harvard Medical School, Boston, MA, USA.

CORRESPONDENCE

M.K. Miller

Genentech

Inc

1 DNA Way

MS 214B

South San Francisco

CA 94044

USA

Fax: 16502254093

E-mail: mkmiller@gene.com

Received:

December 082005

Accepted after revision:

July 072006

SUPPORT STATEMENT

The TENOR study is funded by

Genentech, Inc, and Novartis

Pharmaceuticals Corporation. 


\section{MATERIALS AND METHODS \\ Study design}

The TENOR study was a prospective, 3-yr observational study conducted in the USA in patients with clinician-assessed severe or difficult-to-treat asthma. Patients enrolled in TENOR continued to receive asthma medication and treatment, as indicated by their asthma specialist. There was no specific study intervention. The design and protocol of TENOR were approved by a central institutional review board and, as appropriate, the institutional review board at each site. The TENOR methodology and baseline population characteristics have been described previously [24].

All TENOR subjects were considered by physician evaluation to have severe or difficult-to-treat asthma as a criterion for study entry. Therefore, patients with mild or moderate asthma were eligible if their treating physician considered their asthma "difficult-to-treat" and they met all other inclusion criteria. Patients selected for TENOR received care from their physician or healthcare provider for at least $1 \mathrm{yr}$, and had high use of the healthcare system and/or high medication use in the previous 12 months. High healthcare use in the previous 12 months was defined as two or more unscheduled care visits for asthma or two or more oral corticosteroid bursts (i.e. a short-term increase in steroid therapy to treat an exacerbation of symptoms). High medication use was defined as either currently requiring three or more medications to control asthma, the need for daily high doses of inhaled corticosteroids, or current use of oral prednisone $\geqslant 5 \mathrm{mg} \cdot$ day $^{-1}$. Daily high doses of inhaled corticosteroids were defined by the American Thoracic Society refractory asthma guidelines for adults [25]. Although subjects aged $\geqslant 6$ yrs were eligible for TENOR, for greater homogeneity the current analysis was limited to patients aged $\geqslant 18 \mathrm{yrs}$ at study entry.

\section{METHODS AND ANALYSIS Outcome measures}

In the TENOR study, healthcare use outcomes were assessed every 6 months as a part of regularly scheduled patient interviews and examinations. For this analysis, the primary healthcare use outcomes were ED visit or overnight hospitalisation over the previous 3 months based on subject-elicited survey responses obtained through study coordinator-administered questionnaires; confirmation with patient medical records was not carried out. The primary analysis used visits or overnight hospitalisations reported at 6-month study follow-up. In a secondary analysis, re-testing the model (see below), the outcomes ED visit or overnight hospitalisation reported at 18-month follow-up was studied.

\section{Predictive variables}

The present analytical strategy was to test as wide a scope as possible of potential factors that might contribute to an integrated risk score. Initially, $>200$ candidate baseline variables and new variables derived from those baseline variables were analysed. These candidate variables included demographics, smoking status, medication use (including dose, formulation and frequency), asthma and allergy history, laboratory data (immunoglobulin (Ig)E and pulmonary function tests), and patient-reported outcomes. Actual medication use was collected by study coordinator interview to reflect current use. To collect medication data as accurately and consistently as possible, patients were asked to bring their asthma medications to their study visit. Geographical location (i.e. variable of zip code) was excluded from consideration because of the potential difficulty in generalising specific data obtained from the TENOR population. Physicians' baseline asthma severity and difficult-to-treat assessments were also excluded because this may have been influenced by prior healthcare use, which is likely to be closely related to future healthcare use, the outcome of the analysis. A list of the potential candidate variables is provided in Appendix 1.

To simplify the data that might ultimately be needed to calculate the risk score in actual clinical practice, a priori measures from multi-item questionnaire batteries, such as asthma control based on the Asthma Therapy Assessment Questionnaire (ATAQ) [26] or asthma-related quality-of-life score [27, 28], were excluded. Prior healthcare use was also excluded from the main risk score, in the large part because this potential risk factor was likely to be over-represented in the study group based on TENOR entry criteria. Prior healthcare use variables were addressed in additional sensitivity analyses carried out to evaluate their impact on the test characteristics of the final scoring algorithm (see below).

\section{Development of the TENOR risk score}

An iterative logistic regression modelling approach was used to develop the risk score. By specifying the "stepwise" option on the preliminary logistic regressions, the most highly predictive variable was identified, given the other variables already in the model and, at the same time, the influence of this variable on the other candidate variables already in the model at that stage of testing was estimated. To supplement this computer-driven approach, the variable included for testing at each stage was based on the clinical interpretation of the individual variables and the simplicity of the model. Where there was more than one reasonable alternative variable from which to choose, several were tried one by one in further models. For example, when race/ethnicity was found to be predictive, white/non-white, black/non-black, white/black/ other, white/Hispanic/other and white/black/Hispanic/ other were considered as alternatives.

Once several variables were selected by this algorithm as components of the risk score, a tentative partial risk score was calculated by rounding the regression weights to integers for simplicity of later scoring. Each two-point increase in the risk score was designed to represent an approximate doubling of risk; each one point reflects an increased risk of a factor of approximately 1.41 (square root of 2). For example, the higher risk category of race/ethnicity and of lung function was scored with two points because the odds ratio for that category was close to 2.0. Then the preliminary risk score generated was entered into a logistic regression. Once again, a "stepwise" approach was used to test all of the individual variables for their contribution to the model. If a variable that was part of the preliminary risk score was still statistically significant when added to the model that already included it as part of the preliminary risk score (and possibly additional variables), that was taken as evidence that the incorrect weight had been assigned to that variable. The process of adding variables and testing the resulting risk score continued iteratively until none 
of the candidate variables materially improved the explanatory power of the model.

Iterative testing of the model was begun by considering the demographical and asthma medical history variables and laboratory data. Spirometry measures were assessed using alternative cut-off points that were multiples of $5 \%$ of predicted points. In addition to forced expiratory volume in one second (FEV1), forced vital capacity (FVC) and mean forced expiratory flow between 25 and 75\% of FVC (FEF25$75 \%)$, the ratio of FEV1 to FVC was also evaluated. Each of these was tested separately, given their colinearity. Body mass index $\left(\mathrm{BMI} ; \mathrm{kg} \cdot \mathrm{m}^{-2}\right)$ cut-off points were modelled at intervals of five units. From this intermediate risk score, variables corresponding to major comorbid conditions and asthma-related medications were added. To better understand the impact of medications, the individual medications, including dose and class, were systematically evaluated. Classes of medications were included for clinical coherence and to allow for generalisation to other drugs that were in the same class, but may have been omitted from the TENOR medication list. For missing data, median values were imputed for continuous variables (IgE and spirometry measures) and assumed to be null for the exposure variables.

Points assigned to the individual variables were based on keeping the tool relatively simple (i.e. no fractional points) while capturing most of the information in the variable. For additional simplification, the risk score values were divided into the following three groups: 1) low, 2) medium, and 3) high risk of subsequent ED visit or hospitalisation or both.

\section{Evaluation of TENOR risk score and variants}

After the final TENOR risk score was developed, sensitivity testing was also carried out using two variants of the basic score. The first included the score points derived from the TENOR asthma control/symptoms variables and additional data from Patient Reported Outcome (PRO) questionnaires (risk score with PRO). In the second, the score was re-tested further using healthcare use variables, specifically, prior asthma-related unscheduled office visits, hospitalisations, ED visits and scheduled office visits (risk score with PRO and healthcare use). These risk scores were further evaluated with the missing value imputation by testing only among subjects with complete data.

\section{Internal validation}

Additionally, TENOR longitudinal follow-up data were used to internally validate the risk scores developed from baseline and 6-month follow-up data. In the follow-up validation analysis, the subset of patients with 12- and 18-month follow-up data (internal validation subset) were used to calculate the risk score at month 12 and to predict ED visits or hospitalisation at months 16-18. To assess potential differences between patients in the internal validation and those not, risk scores were calculated from baseline data for patients not part of the internal validation because of insufficient follow-up data (noninternal validation subset), as well as for the internal validation subset of patients.

\section{Statistics}

Although selection of variables and assignment of points were guided by the traditional statistical significance level of $p=0.05$, the goal of the modelling was a simple predictive score, not a formal hypothesis test of the individual factors. Comparisons between populations were carried out using Chisquared tests (for discrete variables) and two-sample t-tests (for continuous variables). Assessment of the explanatory power or discrimination properties for the alternative risk scores (ability of the models to separate patients with different outcomes) was carried out using the c-index, the area under the receiver-operating characteristics curve [29]. A c-index value of 1.0 reflects perfect discrimination, whereas a value of 0.5 reflects no better discrimination than by chance alone.

\section{RESULTS}

Patient characteristics of the analysis population are shown in table 1 . The mean age of the 2,821 patients was $\sim 50 \mathrm{yrs}$, the majority were female $(72 \%)$ and white $(81 \%)$. More than onethird were well-educated $(38 \%$ with college graduate/ advanced degree) and nearly half were employed (48\%) with commercial healthcare coverage $(44 \%)$. Most patients $(56 \%)$ also indicated use of three or more asthma-control medications at baseline.

In total, 1,819 TENOR patients had data available at months 12 and 18 for the internal validation (table 1). Consistent with a survivor effect, the nonvalidation subset patients $(n=1,002)$ differed significantly from the validation patients by age (mean $48.6 \pm 15.17$ for nonvalidation versus $50.2 \pm 14.36$ for validation group, $\mathrm{p}=0.006)$, race/ethnicity $(77.6 \%$ white in nonvalidation versus $82.5 \%$ white in validation group, $\mathrm{p}=0.001$ ) and prior healthcare use $(6 \%$ prior hospitalisation in nonvalidation versus $3.6 \%$ in validation group, $\mathrm{p}=0.004 ; 17.8 \%$ prior $\mathrm{ED}$ in nonvalidation and $11.5 \%$ in validation group, $\mathrm{p}<0.001$ ). Additionally, the internal validation subset results for the PRO and for the PRO and healthcare use variants did not differ substantially from the TENOR risk score (data not shown).

The components entering the final TENOR risk score are shown in table 2. These include: demographics (age, sex, race/ ethnicity); general health status (BMI, diabetes); potential asthma complications or asthma therapy complications (history of pneumonia, history of intubation for asthma, history of cataracts); lung function; and asthma-specific medications (number of prior oral steroid bursts, nebulae ipratropium bromide (Atrovent ${ }$; Boehringer Ingelheim, Ridgefield, CT, USA) use, and current systemic corticosteroid use). The point distribution and proportion of patients within each component category, as well as the proportion of patients with future hospitalisation or ED visits in each component category, is also shown. Overall, $239(8.5 \%)$ of the TENOR subjects studied reported a hospitalisation or an ED visit for asthma at 6-month follow-up. The number of points assigned is positively associated with the percentage of patients with a hospitalisation or an ED visit in that component category. For example, more patients having three or more steroid bursts, compared with patients with no steroid bursts, had more future asthmarelated ED visits or hospitalisations (23 versus 5\%, respectively). The TENOR risk score potentially ranges in value from 0-18. Additional patient-reported outcome components added 


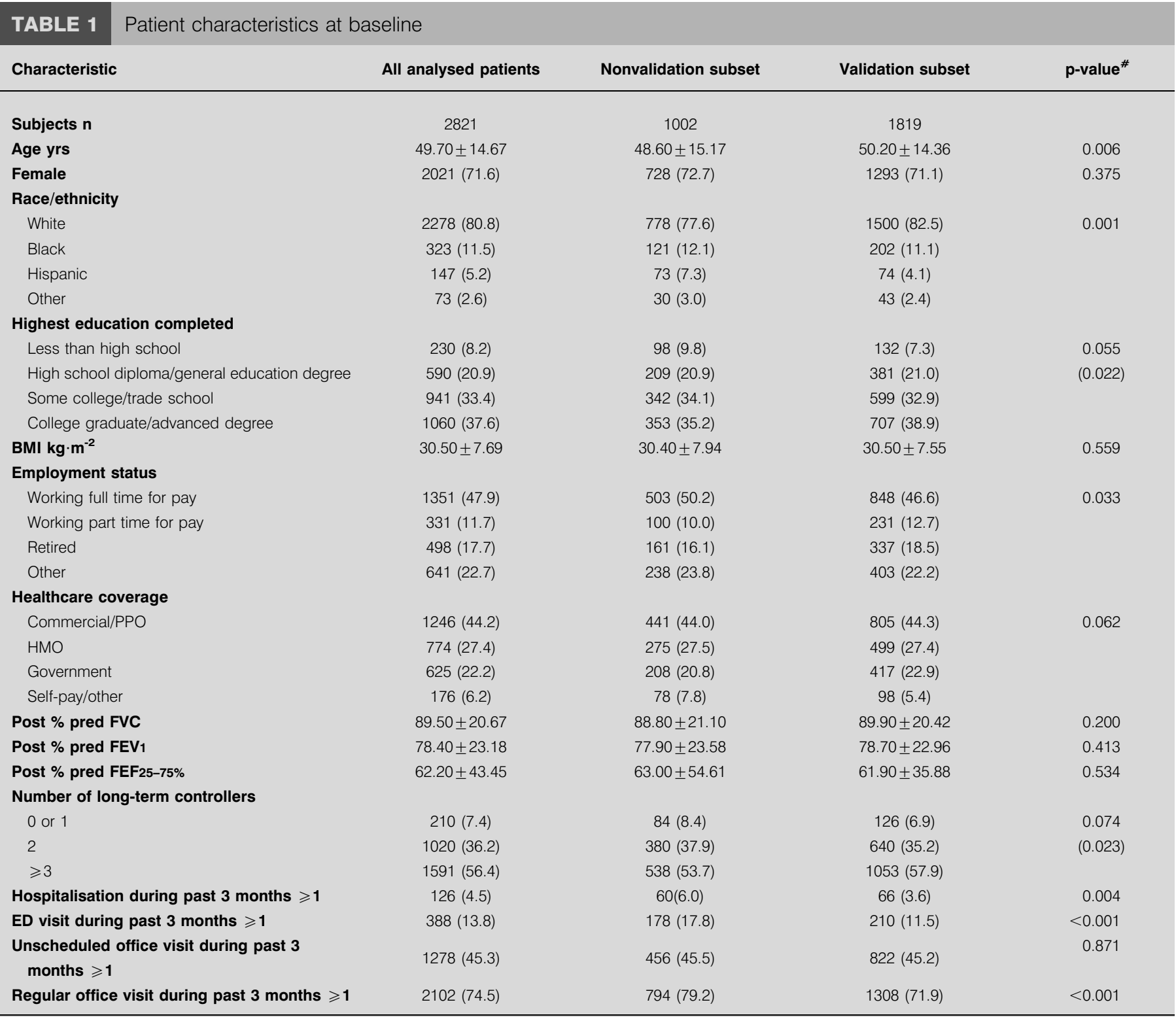

Data are presented as $n(\%)$ or mean \pm sD, unless otherwise indicated. BMI: body mass index; PPO: preferred provider organisation; HMO: health maintenance organisation; \% pred: per cent predicted; FVC: forced vital capacity; FEV1: forced expiratory volume in one second; FEF25-75\%: mean forced expiratory flow between 25 and $75 \%$ of FVC; ED: emergency department. *: comparison is between patients in original population and not in internal validation subset $(n=1,002)$ with patients in internal validation subset $(n=1,819)$. All $p$-values were obtained from calculating Mantel-Haenszel Chi-squared statistic.

four more points, and healthcare use components added an additional 14 points to the total possible score (table 3).

The average values and c-indices of the risk scores and variants for the original population, internal validation subsets and noninternal validation subset are shown in table 4 . The c-index for the risk score of the original population was 0.783 . When the PRO and healthcare use variables are added to the risk score, the mean score values $(6.6 \pm 3.2$ and $8.03 \pm 4.1$, respectively), as well as the c-index (0.798 and 0.816 respectively), increased marginally compared with the risk score calculated without these additional variables. On average, the risk scores were lower for the internal validation subsets than for the original population, reflecting the internal validation's slightly healthier population; however, the c-indices for the internal validation and noninternal validation subsets risk scores were high.

Table 5 shows the TENOR risk score for the original population and internal validation subset divided into three groups, with scores of $0-4$ representing low risk, scores of 5-7 representing moderate risk, and a score of eight or more representing high risk. Point cut-off values were based on percentages of patients with a hospitalisation or an ED visit from the original population analysis. A dramatic increase in the proportion of hospitalisations or ED visits is seen with scores eight or more. The internal validation subset had a 


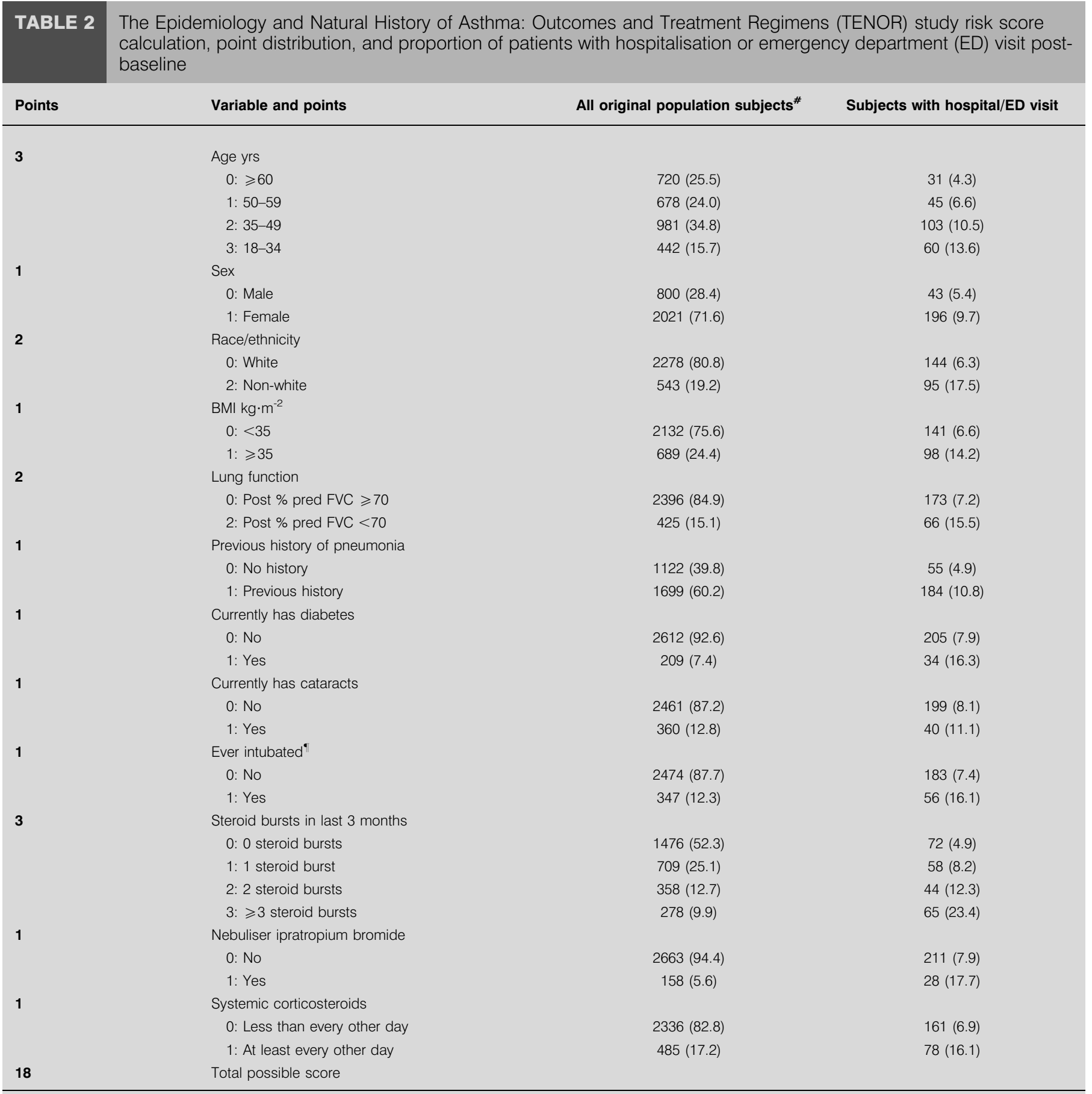

Data are presented as $n$ (\%). BMI: body mass index; \% pred: per cent predicted; FVC: forced vital capacity. ${ }^{\#}: n=2,821 ;$; : ventilator use.

similar overall risk score point distribution to the TENOR risk score when divided into low-, moderate- and high-risk classifications. Figure 1 shows the actual range of the TENOR risk score (0-15) for the original population, as well as for the proportion of patients with a hospitalisation or ED visit reported at 6-month follow-up.

\section{DISCUSSION}

The TENOR risk score is a simple, easy-to-use tool that, in this analysis, was capable of identifying future ED visits or hospitalisations in adult patients with severe or difficult-to-treat asthma enrolled in the TENOR study. As formulated, it does not require input from the patient and could be completely derived from the patient record. The score's multiple components are based on readily available data that would have empirical clinical relevance to healthcare providers. Overall, the risk score performed well not only in the initial iterative testing, but also in an internal validation. A TENOR risk score value of moderate magnitude (5-7 points) reflects a 3.5-fold higher risk of an ED visit or hospitalisation; a high 
TABLE 3 The Epidemiology and Natural History of Asthma: Outcomes and Treatment Regimens (TENOR) study risk score calculation, point distribution and proportion of patients with hospitalisation or emergency department (ED) visit postbaseline for original population using patient reported outcomes and healthcare use variables

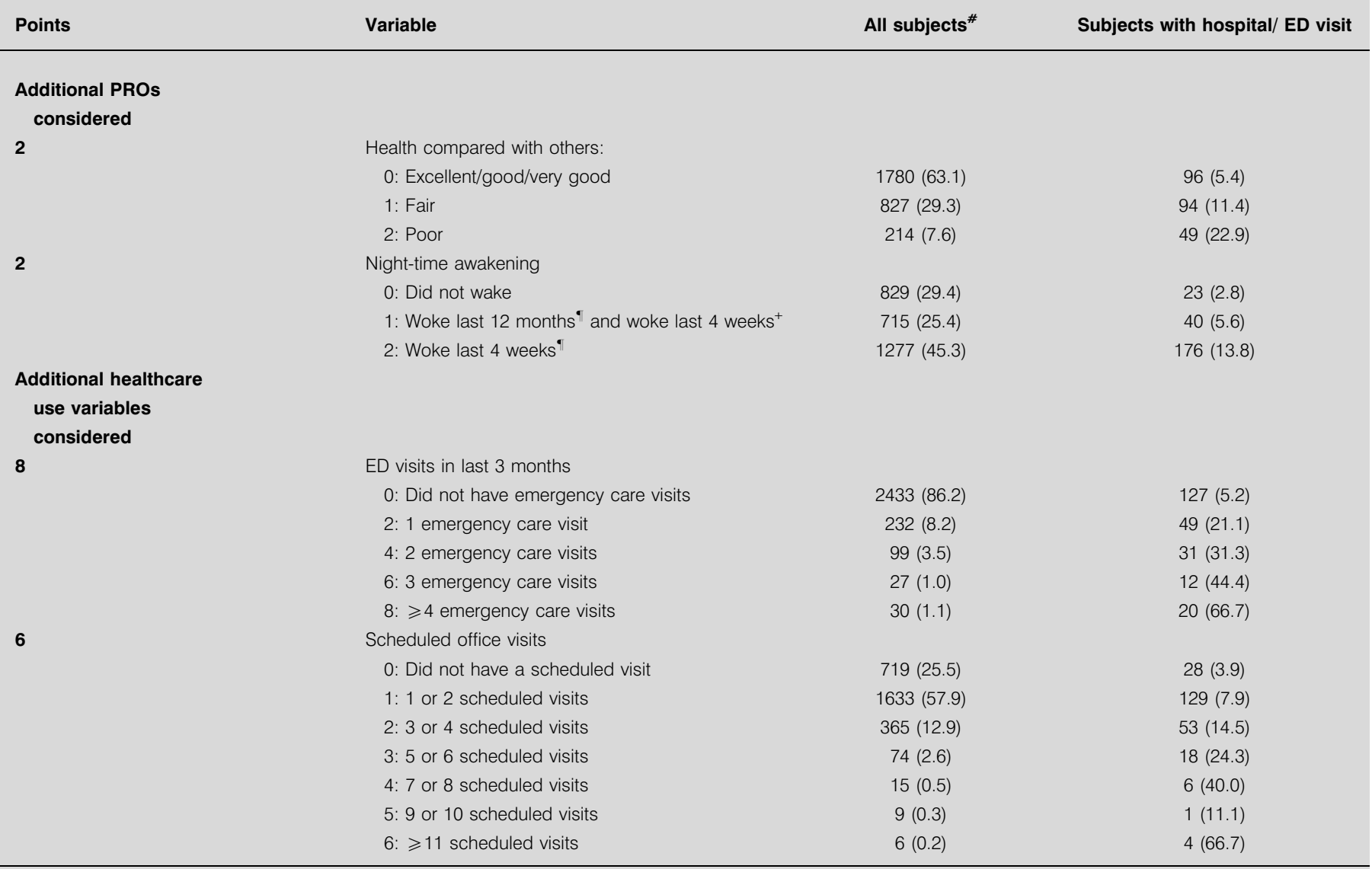

Data are presented as n (\%). PROs: patient reported outcomes. ${ }^{\#}: \mathrm{n}=2,821$; ${ }^{\natural}$ : yes or unsure; ${ }^{+}$; no.

value ( $\geqslant 8$ points) reflects a 12 -fold higher risk compared with patients with low-risk scores (0-4 points). Categorising the risk score into low-, moderate- and high-risk levels adds simplicity with only minimal loss of explanatory power. Although these cut-off values were based on the observed distribution of events at 6-month follow-up, the explanatory

\section{TABLE 4 Descriptive statistics and c-indices for risk scores}

\begin{tabular}{|c|c|c|c|c|c|}
\hline \multirow[t]{2}{*}{ TENOR risk score } & \multirow[t]{2}{*}{$\mathbf{n}$} & \multirow{2}{*}{$\begin{array}{c}\text { Theoretical risk score } \\
\text { range }\end{array}$} & \multirow[t]{2}{*}{ Mean \pm SD } & \multicolumn{2}{|r|}{ c-index } \\
\hline & & & & Full score & Low-medium-high score \\
\hline Original population at baseline & 2821 & $0-18$ & $5.01 \pm 2.45$ & 0.783 & 0.759 \\
\hline Noninternal validation subset at baseline & 1002 & & $5.28 \pm 2.55$ & 0.782 & 0.758 \\
\hline Internal validation subset at baseline & 1819 & & $4.86 \pm 2.39$ & 0.777 & 0.756 \\
\hline Original population at baseline & 2821 & & $6.61 \pm 3.15$ & 0.798 & 0.767 \\
\hline Internal validation subset at month 12 & 1819 & & $5.40 \pm 2.89$ & 0.790 & 0.755 \\
\hline TENOR risk score with PRO and healthcare use & & $0-34$ & & & \\
\hline Original population at baseline & 2821 & & $8.03 \pm 4.14$ & 0.816 & 0.787 \\
\hline Internal validation subset at month 12 & 1819 & & $6.30 \pm 3.58$ & 0.810 & 0.773 \\
\hline
\end{tabular}

TENOR: The Epidemiology and Natural History of Asthma: Outcomes and Treatment Regimens study; PRO: patient reported outcomes. 


\begin{tabular}{|c|c|c|c|c|c|c|c|}
\hline TABLE & \multicolumn{7}{|c|}{$\begin{array}{l}\text { The Epidemiology and Natural History of } \\
\text { Asthma: Outcomes and Treatment Regimens } \\
\text { (TENOR) study risk score by low/medium/high } \\
\text { risk for original and internal validation } \\
\text { populations }\end{array}$} \\
\hline \multirow[t]{3}{*}{ Risk } & \multicolumn{4}{|c|}{ Original population } & \multirow{2}{*}{\multicolumn{3}{|c|}{$\begin{array}{c}\begin{array}{c}\text { Internal validation } \\
\text { subset }\end{array} \\
\text { Hospitalisation/ED }\end{array}$}} \\
\hline & \multirow{2}{*}{$\begin{array}{l}\text { Definition } \\
\text { score }\end{array}$} & \multicolumn{3}{|c|}{ Hospitalisation/ED } & & & \\
\hline & & Total & $\mathbf{n}$ & $\%$ & Total & $\mathbf{n}$ & $\%$ \\
\hline Low & $0-4$ & 1346 & 29 & 2.2 & 1121 & 26 & 2.3 \\
\hline Medium & $5-7$ & 1020 & 91 & 8.9 & 551 & 47 & 8.5 \\
\hline High & $\geqslant 8$ & 455 & 119 & 26.2 & 147 & 43 & 29.3 \\
\hline Total & & 2821 & 239 & 8.5 & 1819 & 116 & 6.4 \\
\hline
\end{tabular}

power using this approach was also similar in the validation analysis.

The internal validity of the TENOR risk score was assessed with prospective testing using additional follow-up data from a subset of the original population. This assessment showed that the TENOR risk score performed well and was highly predictive, and validated its use within the TENOR cohort. While these results are internally valid, they may not be externally valid for other asthma populations.

The TENOR risk score has limitations related to external validity, or generalisability, because the study population does not represent all asthma patients but only severe or difficult-totreat adult asthma patients. The study population may also not completely represent the overall USA population [30,31], with more TENOR patients having access to healthcare and adults educated to at a least high-school level. However, the age and sex distribution of the TENOR population is comparable with other asthma populations $[32,33]$. Since the patients in this analysis are likely to be representative of populations with higher healthcare use for asthma, it is a reasonable cohort in which to study the risk of future ED visits and hospitalisation. In order to address the potential selection effect that the TENOR study entry criteria (as a measure of difficult-to-treat or asthma severity status) could include recent asthma-related healthcare use, healthcare use reported at baseline in the TENOR risk score was not included in the model. Nonetheless, asthma medication use, e.g. oral steroids, was included in the present model which may also be over-represented in TENOR because of the study inclusion criteria. Another potential limitation is the present study's use of ED visits or hospitalisations as the sole surrogate of asthma exacerbation rather than also testing other outcomes, such as steroid bursts or non-ED outpatient physician visits. The use of ED visits or hospitalisations as applied in this analysis, however, is a widely accepted outcome measure reflecting a component of severity or exacerbation [14, 22]. Finally, the present authors recognise that 6 months of follow-up reduced the number of outcome events observed as compared with a longer observation

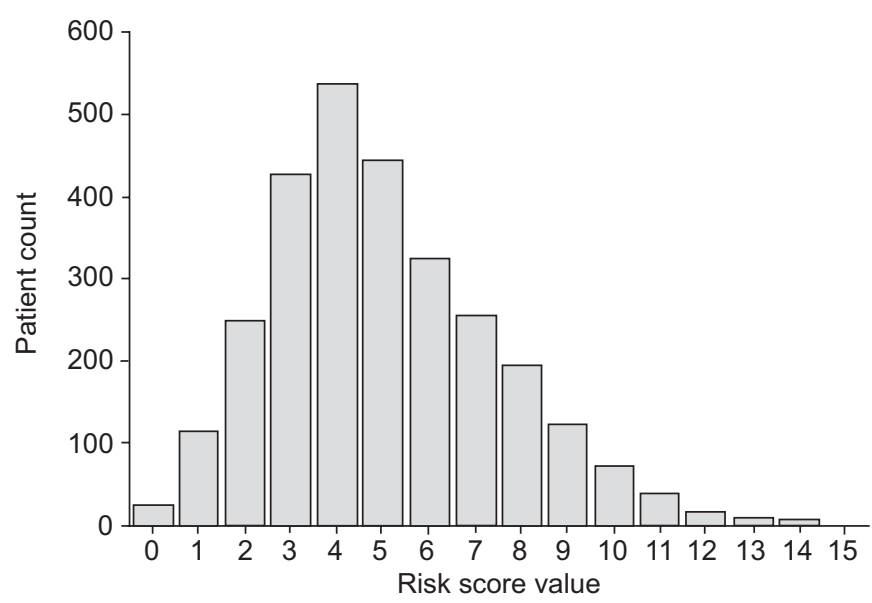

FIGURE 1. Proportions of patients with hospitalisation or emergency department (ED) visit at 4-6 months for The Epidemiology and Natural History of Asthma: Outcomes and Treatment Regimens (TENOR) study risk score. The percentage of those with hospitalisation or ED visit for each risk score value were as follows. Risk score value 0: $0.0 \%$; 1 : 0.0\%; 2: 2.8\%; 3: $0.9 \%$; $4.4 \% ; 5: 7.0 \% ; 6: 9.6 \% ; 7: 11.3 \%$; 8: $19.7 \%$; 9: $16.5 \%$; 10: 33.8\%; 11: 46.2\%; 12: $56.3 \%$; 13: $62.5 \% ; 14: 66.7 \% ; 15$ : $100 \%$.

period, and could limit study power; moreover, this period may not fully account for seasonal variation.

A very large number of patient and clinical variables were considered in developing the risk score. Neither FEV1 nor the FEV1/FVC ratio was as predictive as FVC. Reduction in FVC may have proven to be a powerful variable because in addition to reflecting air trapping in the context of an obstructive ventilatory deficit, it can also be a proxy for restrictive lung physiology associated with obesity, which was indeed relatively common in the present study group. Of note, BMI also entered independently into the final risk score. Although the finding of greater risk associated with obesity is not new, it raises questions about the mechanism of the effect. There has been substantial interest in this topic recently and hypothetical mechanical, inflammatory and hormonal influences have been considered [34]. However, the finding that obesity could be related to steroid use, which also entered into the risk score as steroid bursts as well as systemic corticosteroids. The only nebuliser medication assessed in the TENOR database was ipratropium bromide; albuterol by nebuliser was not specifically ascertained. Thus, it can be presumed that nebuliseradministered ipratropium bromide entered into the score, not because of the importance of nebuliser-administered ipratropium bromide specifically, but because it serves as a proxy for nebuliser use in general, a factor previously shown to be a marker for severe asthma [10].

LIEU et al. [35] attempted to develop a tool to predict healthcare use risk using retrospective demographic and clinical data. Positive predictive values were low (18-26\%), possibly because the analysis was limited to data derived retrospectively from a health maintenance organisation. Other investigators have approached risk prediction with detailed information from patients regarding asthma control [20, 21]. Few predictive indices have been validated prospectively [20]. The indices that 
have been validated prospectively require asking the patient questions to obtain current control status information, which may limit the actual use of the tools.

The TENOR risk score identifies a subset of adult patients with severe or difficult-to-treat asthma at risk of subsequent asthmarelated hospitalisation or ED visits. For example, the risk score of a high-risk patient, who is a non-white ( 2 points), female (1 point), substantially overweight (BMI $>35 \mathrm{~kg} \cdot \mathrm{m}^{-2}$ (1 point)), has medical complications consistent with chronic systemic steroid use (cataracts ( 2 points), diabetes ( 1 point)) and is relatively unresponsive to current corticosteroid medication (three or more steroid bursts in the past 3 months (3 points)), would be nine points. This high-risk patient requires frequent oral corticosteroid bursts and/or chronic use of systemic corticosteroids, as well as nebuliser treatment, a potential marker of multiple medication use. In contrast, the risk score of a low-risk patient, who may be an older ( $\geqslant 60$ yrs ( 0 points $)$ ), white (0 points), male (0 points), with lower BMI (0 points), better lung function ( 0 points) and moderate recent steroid use (two steroid bursts in the past 3 months (2 points)), would be 2 points. Even if a clinician does not formally calculate the TENOR risk score for such patients, recognising the prognostic value of these characteristics may enhance clinical management of patients with severe or difficult-to-treat asthma.

In summary, the ability to predict clinical events is critical for patients, healthcare providers and health systems. A predictive risk score based on clinical variables that can be obtained without patient interaction has been developed. Identification of clinical factors in patients with severe or difficult-to-treat asthma that are associated with future healthcare use may improve asthma patient management and ultimately reduce the burden of disease on the overall healthcare system. The score is ideally suited for adult patients with severe or difficult-to-treat asthma for whom predicting future healthcare needs is clinically relevant. The value of each component of the risk score will be best established by testing it in other populations, including in children and young adults for whom asthma severity is associated with different clinical manifestations, and in other settings such as therapy-intervention studies targeting reduced patient healthcare use or automated medical databases. Additionally, the results of this analysis may be more generalisable to populations with universal healthcare coverage, such as in member countries of the European Union. Although the generalisability of The Epidemiology and Natural History of Asthma: Outcomes and Treatment Regimens risk score is not yet known, calculating the risk score on all asthmatics, including persons who would have fallen outside The Epidemiology and Natural History of Asthma: Outcomes and Treatment Regimens study criteria, could allow potential identification of a subset at higher risk of emergency department visits or hospitalisation, which would be of benefit to clinicians and healthcare systems. Thus, The Epidemiology and Natural History of Asthma: Outcomes and Treatment Regimens risk score tool, although prospectively tested in the target population of The Epidemiology and Natural History of Asthma: Outcomes and Treatment Regimens study, should be further tested in children, adolescents and other adult asthma populations.

\section{APPENDIX 1 COMPLETE LIST OF CANDIDATE VARIABLES \\ Demographics \\ Age}

Sex

Race

Highest grade/year of school completed

Marital status

Employment status

Healthcare coverage

Height

Weight

BMI $\left(\mathrm{kg} \cdot \mathrm{m}^{-2}\right)$

\section{Smoking status}

Ever smoked cigarettes

Currently smoke cigarettes

Current number of cigarettes $\cdot$ day $^{-1}$

Age stopped smoking

Age started smoking

Entire time number of cigarettes $\cdot$ day $^{-1}$

\section{Laboratory data}

$\log \operatorname{IgE}$

Pre \% pred FVC

Post \% pred FVC

Pre \% pred FEV1

Post \% pred FEV1

Pre \% pred FEF25-75\%

Post \% pred FEV25-75\%

Pre \% pred PEFR

Post \% pred PEFR

\section{Asthma history}

Age at asthma diagnosis

Location seen when asthma worsens

Ever used a ventilator tube

Family history

Father had asthma

Mother had asthma

Father had allergic rhinitis

Mother had allergic rhinitis

Father had sinusitis

Mother had sinusitis 
Father had nasal polyps

Mother had nasal polyps

Father had atopic dermatitis

Mother had atopic dermatitis

\section{Personal history}

Ever had chronic bronchitis

Ever had emphysema/chronic obstructive pulmonary disease

Ever had pneumonia

Ever had sneezing or runny or blocked nose without cold or flu

Frequency of stuffy, itchy or runny nose, or watery, itchy eyes

Ever had allergic rhinitis

Ever had itchy rash

Ever had atopic dermatitis

Allergic to insect bites

Breathing difficulty in response to insect bite

Feeling faint in response to insect bite

Nausea in response to insect bite

Fever in response to insect bite

Redness, itching and swelling in response to insect bite

Other response to insect bite

\section{Occupational information}

Ever worked full time for 6 months or more

Ever exposed to dust at work

Type of dust exposure

Ever exposed to gas or chemical fumes at work

Type of gas or chemical fume exposure

Frequently around people who smoke

\section{Residential information}

Currently have pets in home

Number of dogs

Number of cats

Number of birds

Number of hamsters, gerbils or other rodents

Moisture or dampness in house

Visible mould in house

Cockroaches in house in past month

Number of people in household who smoke

\section{Miscellaneous}

Enrolled in an asthma drug-related clinical trial

\section{Comorbid conditions}

History of uticaria/hives

History of rash

History of anaphylaxis

History of other allergic reactions (specify)

Currently has uticaria/hives, rash or anaphylaxis

Multiple sclerosis

Rheumatoid arthritis

Sjogren's syndrome

Systemic lupus erythematosus

Other autoimmune disease

Neoplasia

Diabetes: type 1

Diabetes: type 2

Cataracts

Churg-Strauss syndrome

Erythema multiforme

Parasitic infection

Opportunistic infection (specify)

Osteoporotic fractures

Thrombocytopenia

Heart disease

Vocal cord dysfunction

\section{Medications}

Beclomethasone dipropionate (form and frequency)

Budesonide (form and frequency)

Flunisolide (form and frequency)

Fluticasone (form and frequency)

Triamoinolone acetonide (form and frequency)

Cromolyn (form and frequency)

Nedocromil (form and frequency)

Salmeterol (form and frequency)

Sustained-release albuterol (form and frequency)

Theophylline (form and frequency)

Zafirlukast (form and frequency)

Zileuton (form and frequency)

Advair diskus (form and frequency)

Albuterol (form and frequency)

Albuterol HFA (form and frequency)

Bitolterol (form and frequency) 
Pirbuterol (form and frequency)

Terbutaline (form and frequency)

Ipratropium (form and frequency)

Methylprednisolone (form and frequency)

Prednisolone (form and frequency)

Prednisone (form and frequency)

Montelukast sodium (form and frequency)

\section{Asthma control/symptoms}

Overall health compared to others

Cough, wheeze or other symptoms as a result of exposure

Symptoms due to emotional stress

Symptoms due to exercise

Symptoms due to breathing cold air

Symptoms due to change in weather

Symptoms due to substance abuse

Symptoms due to cold or sinus infection

Symptoms due to personal cigarette smoking

Symptoms due to other people's cigarette smoke

Symptoms due to pollen

Symptoms due to being near animals

Symptoms due to mouldy, dusty or damp places

Symptoms due to breathing dust

Symptoms due to other indoor irritants

Symptoms due to aspirin

\section{Patient-reported questionnaires}

Adult Asthma Therapy Assessment Questionnaire (ATAQ)

Work Productivity and Activity Impairment (WPAI)

Mini Asthma Quality of Life Questionnaire

\section{Healthcare utilisation in last 3 months}

Number of visits to ED

Number of hospitalisations

Total number of days in hospital

Number of steroid bursts

Number of regularly scheduled office visits

Number of unscheduled office visits

Number of missed days from work or school in last 14 days

\section{ACKNOWLEDGEMENTS}

The authors would like to acknowledge that S. Silva of the Ovation Research Group (San Francisco, CA, USA) made significant contributions to the statistical analysis.

\section{REFERENCES}

1 Masoli M, Fabian D, Holt S, Beasley R, for the Global Initiative for Asthma (GINA) Program, The global burden of asthma: executive summary of the GINA Dissemination Committee report. Allergy 2004; 59: 469-478.

2 Mannino DM, Homa DM, Pertowski CA, et al. Surveillance for asthma - United States, 1960-1995. MMWR CDC Surveill Summ 1998; 47: 1-27.

3 National Heart, Lung, and Blood Institute. Morbidity and Mortality: 2002 Chart Book on Cardiovascular, Lung, and Blood Diseases. Bethesda, National Institutes of Health, 2002.

4 Godard P, Chanez P, Siraudin L, Nicoloyannis N, Duru G. Costs of asthma are correlated with severity: a 1-yr prospective study. Eur Respir J 2001; 19: 61-67.

5 Antonicelli L, Bucca C, Neri M, et al. Asthma severity and medical resource utilization. Eur Respir J 2004; 23: 723-729.

6 Serra-Batlles J, Plaza V, Morejon E, Comella A, Brugues J. Costs of asthma according to the degree of severity. Eur Respir J 1998; 12: 1322-1326.

7 National Heart, Lung, and Blood Institute. Morbidity and Mortality: 2004 Chart Book on Cardiovascular, Lung, and Blood Diseases. Bethesda, National Institutes of Health, 2004.

8 Weiss KB, Gergen PJ, Hodgson TA. An economic evaluation of asthma in the United States. N Engl J Med 1992; 326: 862-866.

9 Smith DH, Malone DC, Lawson KA, Okamoto LJ, Battista C, Saunders WB. A national estimate of the economic costs of asthma. Am J Respir Crit Care Med 1997; 156: 787-793.

10 Eisner MD, Katz PP, Yelin EH, Shiboski SC, Blanc PD. Risk factors for hospitalization among adults with asthma: the influence of sociodemographic factors and asthma severity. Respir Res 2001; 2: 53-60.

11 Marcantonio ER, McKean S, Goldfinger M, Kleefield S, Yurkofsky M, Brennan TA. Factors associated with unplanned hospital readmission among patients 65 years of age and older in a Medicare managed care plan. Am J Med 1999; 107: 13-17.

12 Crane J, Pearce N, Burgess C, Woodman K, Robson B, Beasley R. Markers of risk of asthma death or readmission in the 12 months following a hospital admission for asthma. Int J Epidemiol 1992; 21: 737-744.

13 Li D, German D, Lulla S, Thomas RG, Wilson SR. Prospective study of hospitalization for asthma - a preliminary risk factor model. Am J Respir Crit Care Med 1995; 151: 647-655.

14 Adams RJ, Smith BJ, Ruffin RE. Factors associated with hospital admissions and repeat emergency department visits for adults with asthma. Thorax 2000; 55: 566-573.

15 Abramson MJ, Bailey MJ, Couper FJ, et al. Are asthma medications and management related to deaths from asthma? Am J Respir Crit Care Med 2001; 163: 12-18.

16 Prescott E, Lange P, Vestbo J, for the Copenhagen City Heart Study Group, Effect of gender on hospital admissions for asthma and prevalence of self-reported asthma: a prospective study based on a sample of the general population. Thorax 1997; 52: 287-289.

17 Centers for Disease Control. Asthma - United States, 19821992. MMWR Morb Mortal Wkly Rep 1995; 43: 952-955. 
18 Donahue JG, Weiss ST, Livingston JM, Goetsch MA, Greineder DK, Platt R. Inhaled steroids and the risk of hospitalization for asthma. JAMA 1997; 277: 887-891.

19 Brokaw JJ. Asthma in Missouri: recent trends and sociodemographic risk factors. Missouri Med 2001; 98: 67-73.

20 Vollmer WM, Markson LE, O'Connor E, Frazier EA, Berger M, Buist AS. Association of asthma control with health care utilization: a prospective evaluation. Am J Respir Crit Care Med 2002; 165: 195-199.

21 Schatz M, Mosen D, Apter AJ, Zeiger RS, Vollmer WM, Stibolt TB. Relationship of validated psychometric tools to subsequent medical utilization for asthma. J Allergy Clin Immunol 2005; 115: 564-570.

22 Castro M, Zimmermann NA, Crocker S, Bradley J, Leven C, Schechtman KB. Asthma intervention program prevents readmissions in high healthcare users. Am J Respir Crit Care Med 2003; 168: 1095-1099.

23 Bratton DL, Price M, Gavin L, et al. Impact of a multidisciplinary day program on disease and healthcare costs in children and adolescents with severe asthma: a two-year follow-up study. Pediatr Pulmonol 2001; 31: 177-189.

24 Dolan CM, Fraher KE, Bleecker et al, Design and baseline characteristics of The Epidemiology and Natural History of Asthma: Outcomes and Treatment Regimens (TENOR) study: a large cohort of patients with severe or difficult-to-treat asthma. Ann Allergy Asthma Immunol 2004; 92: 32-39.

25 American Thoracic Society. Proceedings of the ATS workshop on refractory asthma: current understanding, recommendations, and unanswered questions. Am J Respir Crit Care Med 2000; 162: 2341-2351.

26 Vollmer WM, Markson LE, O'Connor E, et al. Association of asthma control with health care utilization and quality of life. Am J Respir Crit Care Med 1999; 160: 1647-1652.

27 Juniper EF, Buist AS, Cox FM, Ferrie PJ, King DR. Validation of a standardized version of the Asthma Quality of Life Questionnaire. Chest 1999; 115: 1265-1270.

28 Juniper EF, Guyatt GH, Epstein RS, Ferrie PJ, Jaeschke R, Hiller TK. Evaluation of impairment of health related quality of life in asthma: development of a questionnaire for use in clinical trials. Thorax 1992; 47: 76-83.

29 Hanley JA, McNeil BJ. The meaning and use of the area under a receiver operating characteristic (ROC) curve. Radiology 1982; 143: 29-36.

30 US Census Bureau, 2000 Census of Population and Housing, Summary File 3: Technical Documentation, 2002. Located at: US Census Bureau. Census 2000 Summary File 3, Matrices P37 and PCT2. http://factfinder.census.gov/. Date last accessed: September 25, 2006.

31 DeNavas-Walt C, Proctor B, Mills RJ. Income, poverty, and health insurance coverage in the United States: 2003. US Census Bureau, 2004.

32 Rhodes L, Moorman JE, Redd SC. Sex differences in asthma prevalence and other disease characteristics in eight states. J Asthma 2005; 42: 777-782.

33 Schatz M, Camargo CA Jr. The relationship of sex to asthma prevalence, health care utilization, and medications in a large managed care organization. Ann Allergy Asthma Immunol 2003; 91: 553-558.

34 Schaub B, von Mutius E. Obesity and asthma, what are the links? Curr Opin Allergy Clin Immunol 2005; 5: 185-193.

35 Lieu TA, Capra AM, Quesenberry CP, Mendoza GR, Mazar M. Computer-based models to identify high-risk adults with asthma: is the glass half empty or half full? J Asthma 1999; 36: 359-370. 